\title{
Using accelerometers in the assessment of sarcopenia in older adults attending a day hospital service in Ireland
}

\author{
Kieron Connolly ${ }^{1,2}$, Conal Cunningham ${ }^{3}$, Niamh Murphy ${ }^{1}$, Roman Romero-Ortuno ${ }^{3,4}$, Frances Horgan ${ }^{2}$ \\ 'St James's Hospital, Physiotherapy Department, Ireland; \\ ${ }^{2}$ Royal College of Surgeons in Ireland (RCSI) University of Medicine and Health Sciences, School of Physiotherapy, Ireland; \\ ${ }^{3}$ St James's Hospital, Mercers Institute of Successful Ageing, Ireland; \\ ${ }^{4}$ Trinity College Dublin, Department of Medical Gerontology, Ireland
}

\begin{abstract}
Objectives: The aim of this study was to describe the associations between sarcopenia and variables derived from wrist accelerometry in community-dwelling older adults attending a day hospital service in Ireland. Methods: An observational cross-sectional study was carried out using a consecutive series of older adults attending a day hospital service. Sarcopenia was diagnosed using the latest European Working Group of Sarcopenia in Older People guidelines. Accelerometers were worn by each participant for a 7-day period on their non-dominant wrist. Results: Thirty-eight out of forty-one participants (93\%) met the accelerometer wear time criterion and were included in statistical analyses. Included participants had a mean age of 81.1 years (standard deviation 6.2). Both sarcopenia (Grip) and sarcopenia (Lower limb) were associated with increased time spent in low physical activity and reduced average of Kcals per hour. Only sarcopenia (Lower limb) was associated with increased time in sedentary behaviour as well as reduced number of steps taken in a week. Conclusions: Accelerometer data can be used in an older day hospital population to track physical activity levels and sedentary behaviours. The assessment tool used to assess muscle strength and the cut-off criteria for physical activity behaviour influences the association with sarcopenia.
\end{abstract}

Keywords: Accelerometer, Day Hospital, Frailty, Physical Activity, Sarcopenia

\section{Introduction}

Sarcopenia has been defined as a progressive and generalised skeletal muscle disorder that occurs in ageing and results in reduced muscle mass, reduced muscle strength and poor muscle function ${ }^{1}$. Sarcopenia is associated with various adverse outcomes including mortality, disability, risk of falls and increased cost of care during hospital admissions ${ }^{2-4}$. There are several groups with published definitions and diagnostic procedures for sarcopenia, including the European Working Group of Sarcopenia in Older People (EWGSOP). In 2019, the EWGSOP updated their definition of sarcopenia $\left(\right.$ EWGSOP2) ${ }^{5}$, and their updated definition places a greater emphasis on muscle strength due to its stronger relationship with adverse outcomes ${ }^{6}$. The EWGSOP 2 are the only criteria to define sarcopenia as reduced muscle strength and reduced muscle mass only, while muscle function/physical performance is used to define severity of sarcopenia ${ }^{5}$.

The main factors related to the development of sarcopenia include the effects of ageing, poor nutrition, presence of chronic diseases and physical inactivity ${ }^{7}$. In relation to physical activity (PA) levels, in Ireland two in five men (42\%) and three in five women (59\%) over 75 , report low levels of $\mathrm{PA}^{8}$. The amount of time spent physically active and spent in sedentary behaviour have been linked to increased risk of developing sarcopenia?. Sedentary behaviour (SB) has been linked to poor physical functioning, increased risk of cardiovascular disease as well as all-cause mortality ${ }^{10}$. It is well established that low PA levels are associated with

The authors have no conflict of interest.

Corresponding author: Kieron Connolly, St James's Hospital, Physiotherapy Department, Dublin 8, Ireland

E-mail: kieron.connolly@ucdconnect.ie kconnolly@stjames.ie

Edited by: Charlotte Beaudart

Accepted 20 April 2021 
sarcopenia11-12. A systematic review and meta-analysis including 20 studies by Steffl et al. ${ }^{12}$ highlighted increased levels of PA as a protective factor against sarcopenia in 40,007 individuals, who were predominantly older adults living in the community (mean age 71.7). Sanchez-Sanchez et al. $^{13}$ examined the effects of PA and SB on sarcopenia and the components of sarcopenia. PA and SB were measured using accelerometers and the authors identified an association between increased PA and increased lean body mass. More specifically, using regression models, increased time spent in moderate-vigorous physical activity (MVPA) and a subsequent decrease in time spent in SB was associated with a lower risk of sarcopenia and significantly increased levels of both muscle mass and grip strength $(P<0.001)^{13}$

Exercise (or PA) interventions have the capacity to delay, prevent and reverse the effects of ageing on muscle mass, strength, and frailty by promoting muscle protein anabolism ${ }^{14-15}$. However, it is important to have an accurate measurement of both PA levels and SB in older adults to ensure the efficacy and effectiveness of any intervention. There are numerous ways to assess PA levels ranging from subjective questionnaires to objective data from accelerometers. Unfortunately, subjective questionnaires for PA levels can be hindered by limited recall in older adult populations due to a higher prevalence of cognitive impairment ${ }^{16}$. Accelerometry is one of the most widely used methods to objectively measure PA in a variety of populations and has been validated in older adults ${ }^{17}$.

One essential component of the model of care of older adults in Ireland is the day hospital service ${ }^{18}$. Those attending a day hospital are in receipt of medical care and potentially a multi-disciplinary intervention, due to a change or decline in their health or function. This service provides rapid access to medical and nursing care as well as interdisciplinary and ambulatory care services ${ }^{19}$. The day hospital service remained open even during the COVID-19 pandemic, at a time when many older adults' services in Ireland were curtailed or postponed, potentially delaying a timely Comprehensive Geriatric Assessment (CGA). As part of the COVID-19 strategy In Ireland, national lockdown was implemented, reducing social contacts, outdoor mobility, and common daily activities. The changing of physical activity behaviours and lifestyle has the potential to aid the development of sarcopenia and progression of frailty through psychosocial changes as well as a reduction in quality adjusted years of life $\mathrm{e}^{20,21}$.

To date, there is no information regarding the association between objectively measured accelerometer data on PA, SB levels and sarcopenia in community dwelling older adults attending a day hospital service. The aim of this study was to establish the association between physical accelerometry variables and sarcopenia in community dwelling older adults attending a day hospital service in Ireland.

\section{Materials and Methods}

A consecutive series of older adults attending the Robert Mayne Day Hospital (RMDH) in St James's Hospital (SJH) Dublin, Ireland, were invited to take part in the study between October 2019 and March 2020. Ethical approval was granted for the study by the hospital Research Ethics Committee (reference 2019-10-39[09]). Gatekeepers for the study included the clinical nurse managers (CNMs), RMDH physicians and trainee doctors. All suitable patients were provided with a study patient information leaflet (PIL) by the study gatekeepers. The gatekeepers screened patients in accordance with the study's inclusion and exclusion criteria to determine eligibility for participation.

Older adults (>65 years of age), living in the community, who could sit to stand independently (with or without an aid/ appliance), provide informed consent, understand simple instructions to allow the completion of assessments, and could manipulate a wrist strap physical accelerometer were invited to participate in the study. Individuals who were medically unstable, had a contradiction to Bio-Impedance Analysis (BIA) (e.g. pacemaker, implantable cardioverter device, amputation), had a Clinical Frailty Scale (CFS) of 9 or greater, or had undergone cancer treatment (chemotherapy or radiotherapy) in the last year were excluded. The latter exclusion criterion was implemented to limit the effect of cancer-related cachexia on sarcopenia prevalence.

All potential suitable participants were given at least one week to consider participation in the study before being asked to provide informed consent. All assessments, approximately 30-60 minutes in length, took place in the physiotherapy gym of the RMDH by the principal researcher. Demographic information was collected using the participant's medical chart and electronically using the hospital electronic patient record (EPR) system. Demographic information included participants' age, gender, social deprivation index, past medical history, polypharmacy (>6 medications), living status, presence of home care package, use of meals on wheels service, falls in the last year, hospitalisation in the last year and vitamin D supplementation status (yes or no). The Modified Cumulative Illness Rating Scale (m-CIRS) ${ }^{22}$ was recorded and scoring system developed in the older population by Salvi et al. ${ }^{23}$ was implemented. The m-CIRS was used to assess the severity of comorbidities in this study. The $m$-CIRS grades comorbidities among 13 different organ systems and grades each condition from $\mathrm{O}$ (no problem) to 4 (severely incapacitating or life-threatening conditions) ${ }^{22}$. A higher $\mathrm{m}$-CIRS score is indicative of higher disease burden/severity ${ }^{22}$.

The primary research measure was the diagnosis of sarcopenia and category of sarcopenia using the EWGSOP2 criteria $^{5}$. In the EWGSOP2 criteria, sarcopenia is defined as the presence of low muscle strength and low muscle mass. Probable sarcopenia is defined as reduced muscle strength only (normal muscle mass) ${ }^{5}$. Severe sarcopenia is defined 
as the presence of sarcopenia in combination with reduced physical performance ${ }^{5}$.

Muscle strength was assessed using both handheld grip strength (reduced grip strength $=<27 \mathrm{~kg}$ for men and $<16$ $\mathrm{kg}$ for women) and the Five Times Sit to Stand Test (5STS) (reduced 5STS time $=<15 \mathrm{~s}$ ). Hand-held grip strength assessment (JAMAR Hydraulic Hand dynamometer) procedure was standardised using Roberts et al. $\mathrm{s}^{24}$ recommendations and the strongest measurement from 6 tests ( $x 3$ right and $x 3$ left) was used. The 5-STS test assessment was standardised using the Short Physical Performance Battery (SPPB) instructions ${ }^{25}$.

Muscle mass was assessed using Appendicular Skeletal Mass (ASM) adjusted for height using raw data from BIA (Bodystat Quadscan 4000 Touch, Bodystat, United Kingdom) and validated predictive equations ${ }^{26-27}$. The Sergi et al. ${ }^{16}$ equation predicts ASM from -3.964+(Height[cm $]^{2} /$ Resistance*0.227)+(0.095*Body weight[kg] $)+\left(1.384{ }^{*}\right.$ sex [men $=1$, women $=0])+(0.064 *$ Reactance $)$. The Scafoglieri et al. ${ }^{27}$ equation predicts ASM from $4.957+\left(\right.$ Height $[\mathrm{cm}]^{2}$ / Resistance*0.196)+(0.060*Body weight[kg]) $-(2.554 *$ sex [men=0, women=1]). The cut-offs used to define low muscle mass were set at ASM $<7 \mathrm{~kg} / \mathrm{m}^{2}$ for men and $<6 \mathrm{~kg} / \mathrm{m}^{2}$ for women. All participants were positioned supine on a plinth for the assessment and 4 electrodes were place on each participant's right side (two on dorsal aspect of right hand and two on dorsal aspect of right foot). Fluid intake and bladder voiding were not controlled for in this study. Weight was assessed using a seated SECA 952 scale (kg) (United Kingdom) and measured to the nearest $0.1 \mathrm{~kg}$. Footwear was removed for the assessment. All heavy clothing (coats etc.) were removed prior to the weighing of participants. Height was measured using a SECA 220 stadiometer (United Kingdom) (recorded in $\mathrm{cm}$ ), which was mounted against a wall. Barefoot standing height was measured to the nearest $0.1 \mathrm{~cm}$. The protocol for measuring height was in accordance with Viet and Verschuren ${ }^{28}$.

Physical performance was measured using the Short Physical Performance Battery (SPPB) $(<8 / 12)^{25}$ and/or the Timed Up and Go Test (TUG) (>2Os) ${ }^{29}$ as per the EWGSOP2 criteria ${ }^{5}$.

An individual's risk of sarcopenia was assessed using the SARC-F questionnaire (Strength, Assistance with walking, Rise from a chair, Climb stairs and Falls), where a score of $>4$ was indicative of a risk of developing sarcopenia ${ }^{30}$. Nutritional status was assessed using the modified Mini Nutritional Assessment- Short Form (MNA-SF). An individual is classified as malnourished if scores of $<7$, risk of malnutrition 8-11 and well-nourished/normal if scores $>12^{31}$.

Cognition was assessed using the Quick Mild Cognitive Impairment Screen (QMCI). The components of the $\mathrm{QMCl}$ include orientation $(/ 10)$, word registration $(/ 5)$, clock drawing $(/ 15)$, delayed recall (/20), verbal fluency $(/ 20)$ and logical memory $(/ 30)^{32}$. These areas are scored differently, with a total QMCl score being totalled out of 100 , with
$<62 / 100$ being predictive of mild cognitive impairment $(\mathrm{MCl})$ or dementia ${ }^{32}$.

Frailty was assessed using the Clinical Frailty Scale (CFS) ${ }^{33}$ and the SHARE Frailty Instrument for primary care $(\mathrm{SFI})^{34}$. The SFI is based on a modified phenotypic approach and includes two web-based frailty calculators for each gender ${ }^{34}$. Both calculators are freely accessible on BMC Geriatrics (http://www.biomedcentral.com/ 1471-2318/10/57/ additional). Each participant's SFI scores were inputted into the calculators to determine a frailty status ${ }^{34}$.

Physical activity levels were measured, subjectively, using the International Physical Activity Questionnaire for Elderly (IPAQ-E). The IPAQ-E was scored using the IPAQ scoring protocol ( ${ }^{35}$, https://sites.google.com/site/theipaq/home). When scoring the questionnaire, metabolic equivalents (METS) per week were calculated for walking, moderate and vigorous physical activity (PA) while time spent sitting per week (mins) was also documented. For walking the MET 3.3 was used, for moderate and vigorous activity, the METS 4.0 and 8.0 were used as per the scoring protocol ${ }^{35}$. An individual was classified as having low PA levels if their weekly METS fell below 600 a week, moderate PA levels were classified as METS of 601-2999 a week while high PA levels were classified as $>3000$ METS a week ${ }^{35}$.

Actigraph xGT3-BT Accelerometers (Actigraph, United States of America) were used to assess PA levels objectively. Due to limited resources, every third participant was prescribed a physical accelerometer. All accelerometers were worn on the participant's non-dominant wrist for a 7-day period. Wearing an accelerometer on the wrist has been shown to increase compliance/adherence to wearing the accelerometer and can be worn to bed at night compared to hip worn devices ${ }^{17,36-38}$. Wrist worn accelerometers have been shown to estimate wear time more accurately, compared to the hip worn device ${ }^{36-37}$. Another benefit of placing the accelerometer at the wrist is that in older adults, it captures common ADL performed tasks, portraying a complete picture of daily activity ${ }^{38}$.

Due to resource limitations, not all participants could be prescribed an accelerometer. Due to this and to avoid selection bias, every third participant recruited was invited to wear an accelerometer. Participants were instructed to remove the accelerometer when showering or bathing and encouraged to keep on at night but could remove it if it was uncomfortable.

Each participant with an accelerometer was contacted by phone on day 3 and day 6 of wearing the accelerometer to ensure adherence and return of the accelerometer to the RMDH. The phone call consisted of 4 questions that revolved around any issues regarding the accelerometer and adherence. After the seven-day period, each participant returned the accelerometer to the RMDH or at their following appointment.

Wear Time validation function, in the Actigraph software using the Choi algorithm, was used to identify 


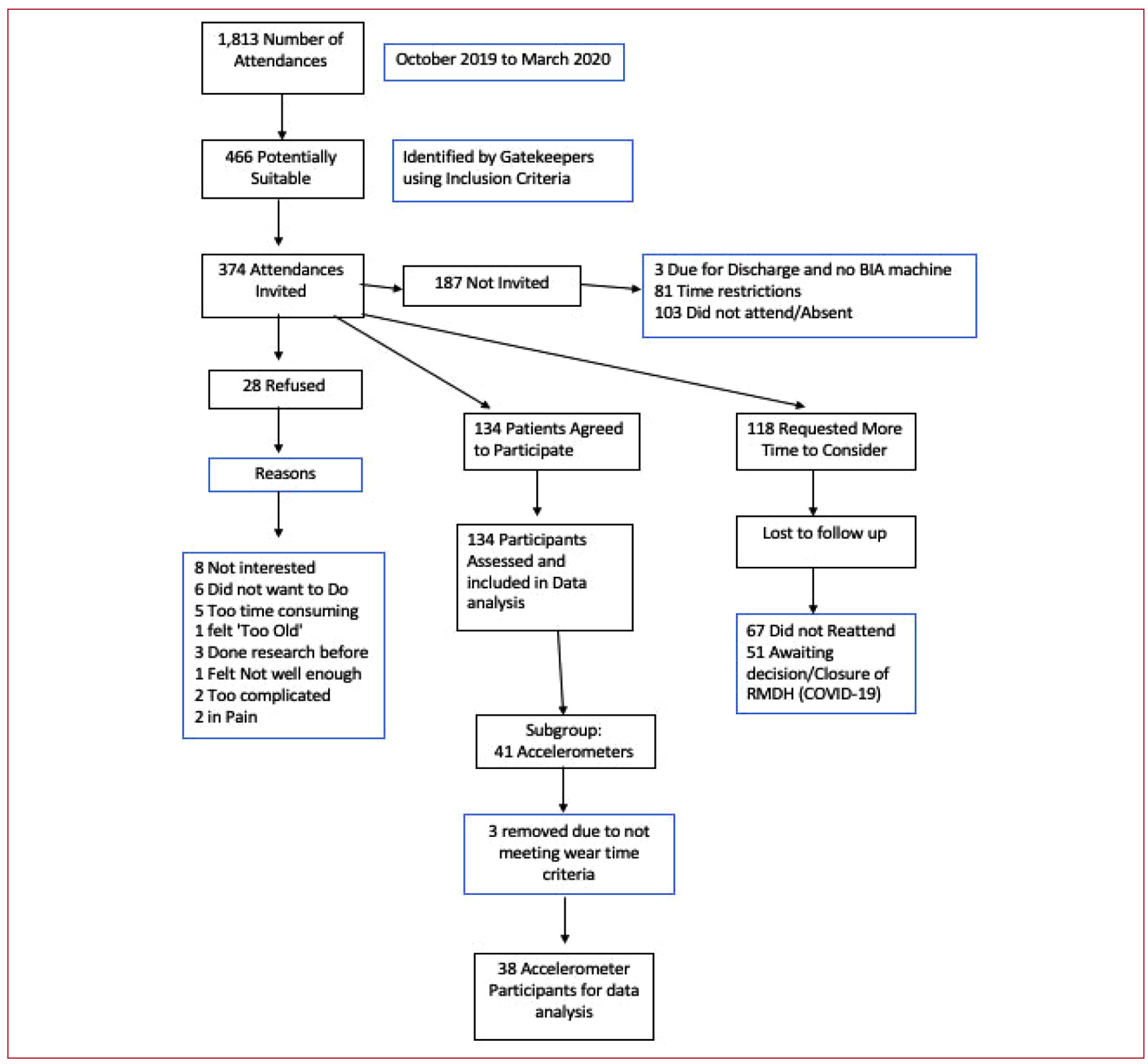

Figure 1. Flow of participants in the study $(\mathrm{N}=134)$. * The number of attendances to the RMDH from October 2019 to March 2020 was 1813 , this number was determined by calculating the number of patients booked in for the day hospital each day. The study was conducted in a busy day hospital clinical environment where the gatekeepers who were members of the clinical team were also asked to screen for eligible study participants. The specific reasons for exclusion were not recorded but would have included age, excessive frailty, and severe cognitive impairment.

and remove participants that did not meet the wear time criteria. All participants with less than 10 hours of wear time for at least 4 days were not included in data analysis. Accelerometer data was downloaded at 1s Epochs, which enabled reintegration as needed for PA criteria. A full description of accelerometer variables can be found in Appendix 1. For determining SB and PA levels, two different reference datasets were used, both were based on vector magnitude, counts per minute on 60s Epochs). The first PA activity criteria was the 'Freedson Adult VM3 2011', which was developed using healthy older adults on a treadmill ${ }^{39}$. This criterion ranges from sedentary to very vigorous activity using vector magnitude counts per minute. The criterion is as follows: 0-99=Sedentary; 100-2689=Light; 2690-6166=Moderate; 6167-9642=Vigorous; >9643= Very Vigorous. The other criterion adopted was that of Sanchez-Sanchez et al. ${ }^{13}$, which is developed from older adults. The criterion ranges from SB (0-173); Low PA (1741924) and MVPA (>1925) $)^{13}$. 


\begin{tabular}{|c|c|c|}
\hline Variable & & $N /(\%)$ \\
\hline Age years, mean (SD) & Range 65-98 & $81.7(7.1)$ \\
\hline Gender & $\begin{array}{c}\text { Male } \\
\text { Female }\end{array}$ & $\begin{array}{l}52(39) \\
82(61)\end{array}$ \\
\hline Living status & $\begin{array}{l}\text { Lived alone } \\
\text { Lived with someone }\end{array}$ & $\begin{array}{l}60(45) \\
74(55)\end{array}$ \\
\hline Supports & $\begin{array}{l}\text { HCP } \\
\text { MOW }\end{array}$ & $\begin{array}{l}20(15) \\
9(6.7)\end{array}$ \\
\hline Reason for referral to RMDH & $\begin{array}{l}\text { Outpatients } \\
\text { Recent inpatient stay } \\
\text { GP referral } \\
\text { Recent attendance at ED }\end{array}$ & $\begin{array}{c}79(59) \\
42(31) \\
3(2) \\
10(8)\end{array}$ \\
\hline Social Deprivation Index living area* & $\begin{array}{l}\text { 'marginally below average' } \\
\text { 'marginally above average' } \\
\text { 'disadvantaged/very disadvantaged' } \\
\text { 'affluent/very affluent' }\end{array}$ & $\begin{array}{l}54(40) \\
40(30) \\
14(10) \\
26(20)\end{array}$ \\
\hline Comorbidities $>6$ & & $82(61)$ \\
\hline Comorbidity details & $\begin{array}{c}\text { Rheumatology } \\
\text { Lung Disease } \\
\text { Cancer History } \\
\text { Osteoporosis/Osteopenia } \\
\text { Chronic Kidney Disease }\end{array}$ & $\begin{array}{l}12(9) \\
41(31) \\
32(24) \\
41(31) \\
23(17)\end{array}$ \\
\hline Polypharmacy & & $85(64)$ \\
\hline
\end{tabular}

Table 1. Description of the study participants $(N=134)$.

\section{Statistical Analysis}

The normality of data was assessed using the Kolmogorov-Smirnov (KS) test for groups with a sample size greater than 50 , with $p$ value being $>0.05$, indicative of normal distribution of data. The Shapiro Wilks (SW) test was used to test the distribution of data for groups with sample size less than 50 , with $p$ value being $>0.05$ indicative of normal distribution of data.

The Actigraph software v5.7.4 and SPSS v26 (IBM Corp) were used for data analysis. Pearson's R (Normally distributed data) and Spearman's Rho Correlation Coefficient (Non-Parametric data) tests were used to assess the strength of correlation between variables. A correlation co-efficient above 0.6 was considered strong if the correlation was between 0.3 and 0.59 it was considered as moderate and a mild correlation was considered less than 0.3. Bivariate analysis was completed using four different tests, depending on the type of variable and the distribution of said variable. For normally distributed data (continuous variables), Independent T-tests were used to compare sarcopenia groups. In non-parametric data (continuous variables), the Mann Whitney $U$ test was used to determine significant differences between groups. To be determined significant, the $p$ value had to be less than 0.05 .

\section{Results}

Participant recruitment was completed over a sixmonth period (October 2019 to March 2020), recruitment ceased prematurely in March 2020 due to the COVID-19 pandemic. The final sample was 134 participants. Of the 134 participants, forty-one $(\mathrm{N}=31 \%)$ participants consented to wear an accelerometer. Three participants with accelerometer data were removed from data analysis due to failure to meet wear time criteria (10 hours for at least 4 days). The flow of participants in the study is shown in Figure 1. Table 1 provides a description of all study participants in relation to demographics.

\section{Description of Accelerometer Subgroup}

Sixty-three percent $(\mathrm{N}=24)$ of the accelerometer group were female and $37 \%$ were male $(\mathrm{N}=14)$. The participants in the accelerometer group had a mean age of $81.1( \pm S D=6.2)$ and mean $\mathrm{Hb}$ levels of $12.2 \mathrm{~g} / \mathrm{dl}( \pm \mathrm{SD}=1.6)$. Using the IPAQ-E to measure PA levels, $44 \%(N=18)$ of the accelerometer group were categorised into the low PA category. When compared to the main study group, the accelerometer subgroup had a significantly higher cognitive score (QMCI), were less frail (CFS), had a lower BMI and performed better in physical performance tests (SPPB and TUG) compared to 


\begin{tabular}{|c|c|c|c|c|c|c|}
\hline Variable & Accelerometer & $\mathbf{N}$ & Mean & SD & $\mathbf{T}$ & $\mathbf{P}$ \\
\hline \multirow[t]{2}{*}{ Age } & No & 93 & 81.9 & 7.6 & \multirow{2}{*}{0.398} & \multirow{2}{*}{0.691} \\
\hline & Yes & 38 & 81.3 & 5.9 & & \\
\hline \multirow[t]{2}{*}{ BMI $\left(k g / m^{2}\right)$} & No & 90 & 27.3 & 5.3 & \multirow{2}{*}{2.28} & \multirow{2}{*}{$0.024^{*}$} \\
\hline & Yes & 38 & 25 & 4.9 & & \\
\hline \multirow[t]{2}{*}{ QMCl_Total } & No & 90 & 48.3 & 14.7 & \multirow{2}{*}{-3.03} & \multirow{2}{*}{$0.003^{*}$} \\
\hline & Yes & 38 & 56.8 & 14.2 & & \\
\hline Variable & Accelerometer & $\mathbf{N}$ & Median & IQR & $\mathbf{z}$ & $\mathbf{P}$ \\
\hline \multirow[t]{2}{*}{$\mathrm{CC}(\mathrm{cm})$} & No & 89 & 35 & 6 & \multirow{2}{*}{-2.05} & \multirow{2}{*}{$0.04^{*}$} \\
\hline & Yes & 38 & 33 & 4 & & \\
\hline \multirow[t]{2}{*}{ SPPB Total } & No & 90 & 9 & 5 & \multirow{2}{*}{-2.46} & \multirow{2}{*}{$0.014^{*}$} \\
\hline & Yes & 38 & 9 & 5 & & \\
\hline \multirow[t]{2}{*}{ TUG_best (s) } & No & 88 & 14.9 & 10.61 & \multirow{2}{*}{-2.81} & \multirow{2}{*}{$0.005^{*}$} \\
\hline & Yes & 38 & 10.7 & 7.3 & & \\
\hline \multirow[t]{2}{*}{ CFS } & No & 92 & 5 & 2 & \multirow{2}{*}{-3.39} & \multirow{2}{*}{$0.001^{*}$} \\
\hline & Yes & 38 & 4 & 2 & & \\
\hline \multicolumn{3}{|c|}{ Variable } & Test & Value & Phi & $\mathbf{P}$ \\
\hline \multicolumn{3}{|c|}{ Vit D supplement } & Pearson Chi-Square & 4.01 & -0.17 & $0.045^{*}$ \\
\hline \multicolumn{3}{|c|}{ Age Group } & Pearson Chi-Square & 4.68 & -0.2 & $0.03^{*}$ \\
\hline \multicolumn{3}{|c|}{ Gender } & Pearson Chi-Square & 0.001 & -0.003 & 0.973 \\
\hline \multicolumn{3}{|c|}{ Poor Physical Performance SPPB <8 } & Pearson Chi-Square & 6.29 & -.22 & .01 * \\
\hline \multicolumn{3}{|c|}{ Poor Physical Performance TUG >20s } & Pearson Chi-Square & .84 & -.08 & .36 \\
\hline \multicolumn{7}{|c|}{ 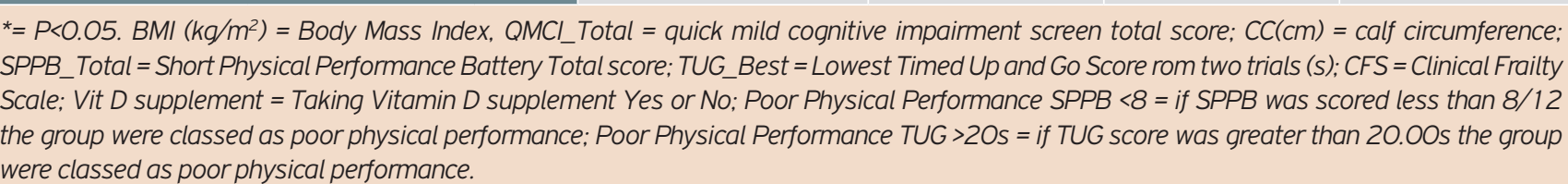 } \\
\hline
\end{tabular}

Table 2. Comparison between Accelerometer and Non-Accelerometer group.

the non-accelerometer participants (Table 2). A description of the whole group and accelerometer group, in relation to anthropometric, physical performance, frailty and cognition can be seen in Table 3.

There were also significant differences in relation to age ( $<85$ vs $>85$ ). Accelerometer subgroup were younger (Pearson Chi-Square 4.68, $\mathrm{P}=0.03, \mathrm{Phi}=-0.2$ ) and taking a vitamin D supplement (Pearson Chi Square: 4.01, $P=0.45$, $\mathrm{Phi}=-0.17$ ). However, there was no difference between the two groups in relation to the vitamin $D$ levels. Using available vitamin $D$ levels, $56 \%(\mathrm{~N}=20)$ of the accelerometer group had normal vitamin $D$ levels compared to $71 \%(\mathrm{~N}=56)$ of the non-accelerometer group (Pearson Chi Square $=2.593$, $\mathrm{Df}=1, \mathrm{P}=0.107$ ). There was no difference in the prevalence of sarcopenia between the accelerometer and nonaccelerometer group, irrespective of how muscle strength was measured. The accelerometer group had a sarcopenia
(Grip) prevalence of $34 \%(\mathrm{~N}=14)$ compared to $23 \%$ $(\mathrm{N}=21)$ in the non-accelerometer group (Pearson Chi Square $\mathrm{P}=0.182$ ). The accelerometer group had a sarcopenia (LL) prevalence of $44 \%(\mathrm{~N}=18)$ compared to $34 \%$ in the nonaccelerometer group ( $\mathrm{N}=31$ ) (Pearson Chi Square $\mathrm{P}=.300$ ).

\section{Accelerometer Variables}

After removing the outliers $(\mathrm{N}=3)$, the median wear time was 156.5 hours (IQR: 54.1 ), which equates to $92 \%$ of wear time over a 7-day period or 6.5 days of wear time. Non-wear time averaged at a median of 13.8 hours (IQR: 59), which equates to less than one full day of non-wear time.

Two different sets of cut-points were used to analyse the breakdown of SB and PA in the accelerometer group. Using the 'Freedson Adult VM3 2011' cut-offs from the Actigraph software, the accelerometer group spent 


\begin{tabular}{|c|c|c|c|c|}
\hline \multirow[b]{2}{*}{ Variable } & \multicolumn{2}{|c|}{ Whole Group $(n=134)$} & \multicolumn{2}{|c|}{ Accelerometer Group ( $n=38)$} \\
\hline & Median & IQR & Mean & \pm SD \\
\hline m-CIRS & 9.4 & 5 & 8.2 & 4.1 \\
\hline Height (cm) & 159.5 & 15.5 & 161.2 & 10 \\
\hline Weight (kg) & 66.7 & 24.2 & 65.5 & 16 \\
\hline Best Grip (kg) & 19 & 12 & 21.2 & 8.5 \\
\hline QMCI & 50.8 & 22.5 & 56.8 & 14.2 \\
\hline SFI & 2.4 & 2.3 & 1.7 & 2.2 \\
\hline Variable & Median & IQR & Median & IQR \\
\hline Gait speed_1 (s) & 4.3 & 2.3 & 3.6 & 2.6 \\
\hline Gait speed_2 (s) & 3.9 & 2 & 3.4 & 1.7 \\
\hline 5-STS_Time (s) & 14.9 & 9.2 & 14.17 & 5.9 \\
\hline SPPB_Total (/12) & 7 & 5 & 9 & 5 \\
\hline TUG_Best (s) & 13.9 & 10.9 & 10.7 & 7.3 \\
\hline CFS & 4 & 2 & 4 & 2 \\
\hline Modified MNA-SF & 11 & 3 & 11 & 3 \\
\hline Sitting mins/Day & 360 & 197.5 & 360 & 360 \\
\hline WalkingMETS/w & 338.3 & 858 & 462 & 1237.5 \\
\hline ModMETS/w & 604.5 & 530 & 40 & 840 \\
\hline VigMETS/w & O & 0 & 0 & 0 \\
\hline TotalMETS/w & 604.5 & 1594.5 & 786.5 & 1844 \\
\hline \multicolumn{5}{|c|}{ 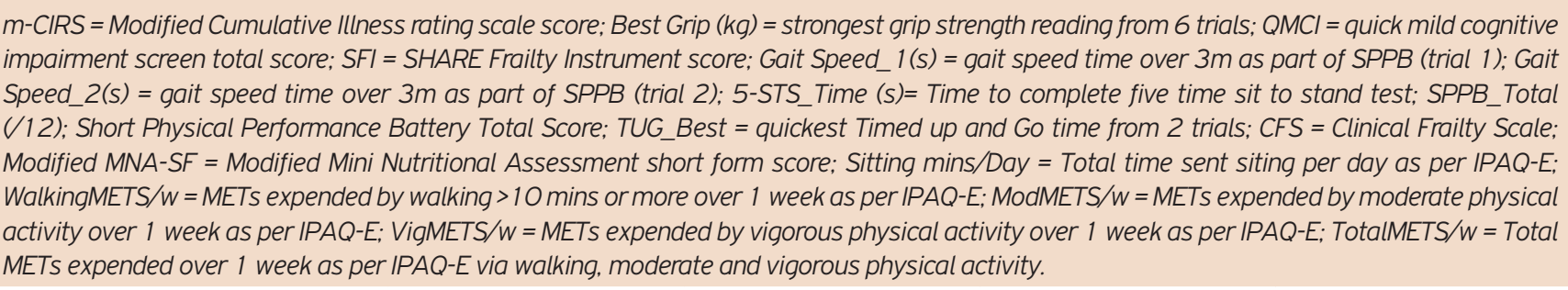 } \\
\hline
\end{tabular}

Table 3. Description of the Whole group ( $N=134)$ and Accelerometer subgroup ( $N=38)$.

$85.4 \%( \pm S D=6.3 \%)$ of wear time in light $P A$ and the remaining $14.6 \%( \pm S D=6.3 \%)$ in moderate $P A$. This represents a median time of 129.8 hours (IQR: 51 ) in light PA and 18.42 hours (IQR: 16.2) in moderate PA over the wear time. The accelerometer group failed to reach the vigorous or very vigorous exercise intensity as part of the 'Freedson Adult VM3 2011' cut-offs. Per day, the average MVPA time equated to a median 2.2 hours (IQR: 2.06). The mean steps per minute were $6.4( \pm S D=3)$ and the median total step counts over the total wear time were 46,373.5 (IQR: 42,013.8). Using the Sanchez-Sanchez et al. (2019) cut-offs, the accelerometer group spent a median of $71 \%$ (IQR: $15.5 \%$ ) of their wear time in SB, $9 \%$ (IQR: $3.6 \%)$ in low PA and a mean $21 \%( \pm S D=8.1 \%)$ in MVPA. In relation to the differences between the Freedson
Adult VM3 2011 and Sanchez-Sanchez et al. (2019) cut-offs in MVPA, there is approximately a 7.3-8.1-hour difference between the two classification criteria.

The m-CIRS was negatively correlated with PA levels, specifically with number of steps taken (Spearman $\mathrm{R}=-.41, \mathrm{p}=0.013$ ), percentage of time spent in MVPA (Freedson Adult VM3 2011 ) (Spearman R=-.33, p=0.05) and percentage of time spent in MVPA (SanchezOSanchez et al. 2019) (Spearman $R=-.35, p=0.04$ ). Vitamin D levels were moderately correlated with amount of Vig METS achieved each week (Spearman $\mathrm{R}=.363, \mathrm{p}=0.035$ ). CRP was moderately correlated with SB, indicating a higher CRP levels would increase the amount of time spent in SB and reduce the amount of time spent physically active $(p<0.05)$. 


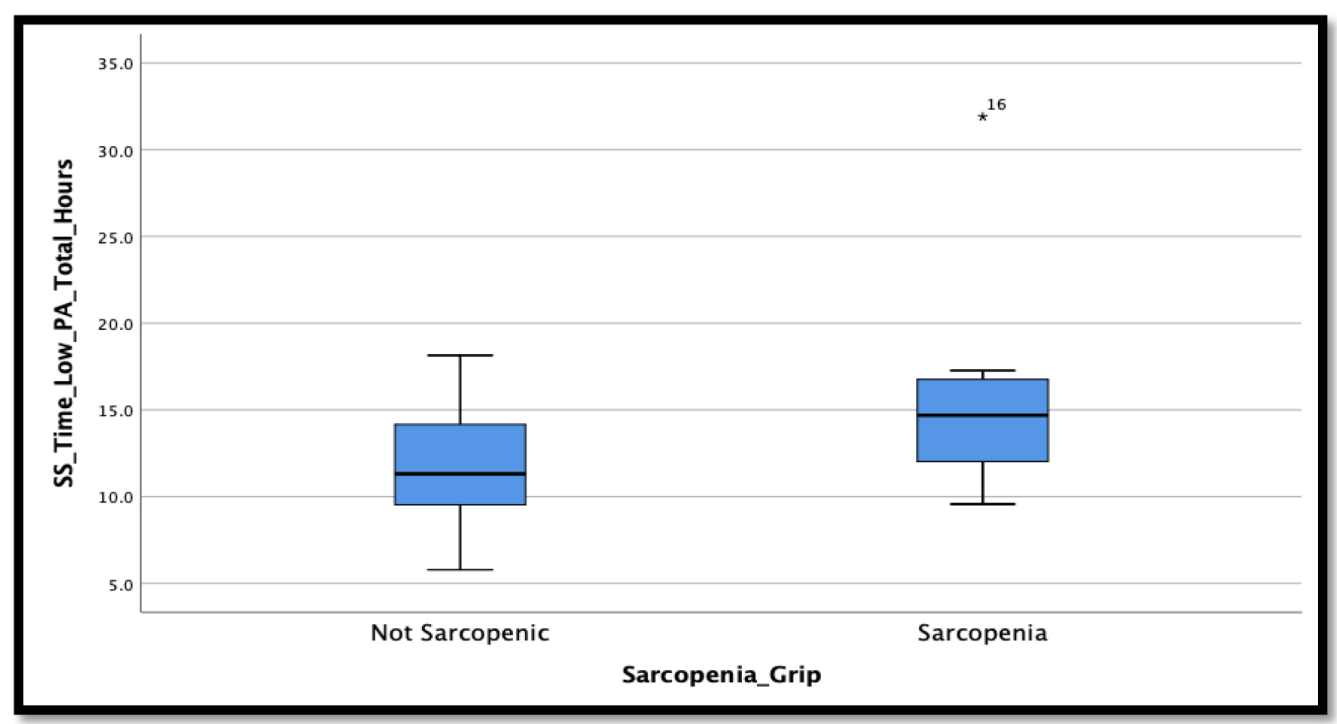

$\mathrm{b}$

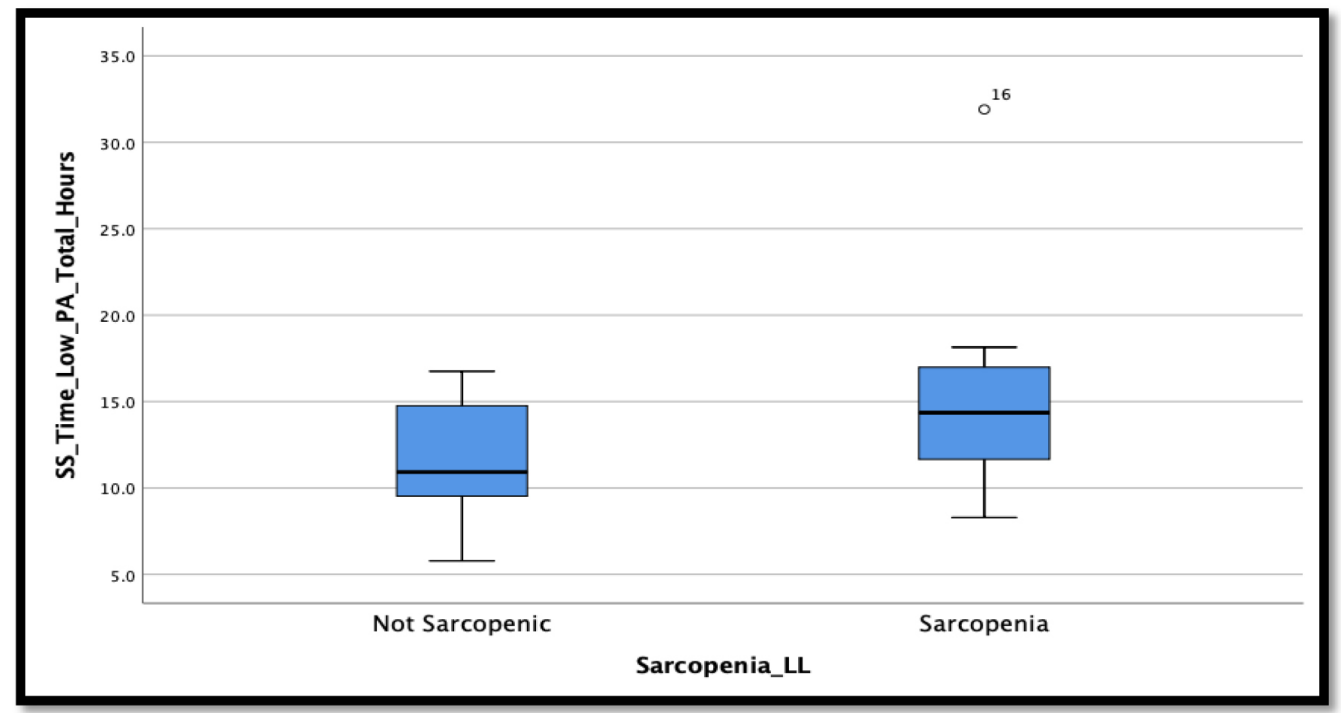

Figure 2. (a) Total hours spent in low PA and sarcopenia (Grip) (b) Total hours spent in low PA and sarcopenia (LL). SS_Time_Low_PA_Total_Hours = Time spent (hours) in low physical activity using Sanchez-Sanchez et al. (2019) cut-off criteria. Sarcopenia_Grip = sarcopenia defined as low muscle strength (reduced grip strength) and reduced muscle mass (bio-impedance analysis). Sarcopenia_LL = sarcopenia defined as low muscle strength (reduced lower limb strength) and reduced muscle mass (bio-impedance analysis).

\section{Sarcopenia and Accelerometer Data}

Sarcopenia (Grip) and Sarcopenia (LL) were both positively associated with the amount of time spent in low PA (Total Hours and Total mins) $(\mathrm{Z}=-2.17-2.31, \mathrm{p}=0.03)$ (Figure $2 \mathrm{a} \&$ b) and negatively associated with the average kcals per hour (sarcopenia mean=31.1-29.2 and non-sarcopenia mean= 44.1-47.3, $Z=2.3-2.7, p=0.001-0.029$ ). Only sarcopenia (LL) was associated with a reduced number of steps per minute $(p=0.015)$, increased amount of time in sedentary bouts per day and over the course of 7 days compared to the non-sarcopenic group ( $p=0.021-0.029)$. Using Freedson's
Adult VM3 2011 cut-offs for light, moderate and vigorous activity, only the time spent in light PA was significantly different between the sarcopenia (LL) participants and nonsarcopenic participants $(p=0.009)$, which was not found in sarcopenia (Grip). Using the Sanchez-Sanchez et al. (2019) cut-offs, only sarcopenia (LL) was associated with time spent in $S B(Z=-2.1, p=0.04)$.

\section{Discussion}

In the small subgroup prescribed an accelerometer, the use of a wrist worn physical accelerometer was feasible 
and practical in tracking PA and SB variables in older adults attending a day hospital. In the accelerometer sub-group, participants spent approximately $80-85.4 \%$ of their week in SB/light PA, depending on the cut-off scores used. The remaining time was spent in MVPA. In relation to sitting time, the accelerometer group spent a median 6 hours ( 360 mins) sitting per day as per the IPAQ-E. An increased amount of time spent in low PA and decreased number of kcals expended per hour were associated with both, sarcopenia (Grip) and sarcopenia (LL). Sarcopenia (LL) was also associated with increased amount of time spent in SB and a reduced number of steps over a 7-day period.

The accelerometer group were less active compared to healthy older adults living in the community across Ireland, when using a subjective measurement. In the TILDA study ${ }^{40}$, using the IPAQ, older adults spent a mean time of $296( \pm \mathrm{SD}=157)$ minutes sitting a day (equals 4.9 hours) compared to 360 mins ( 6 hours) in the accelerometer group (per week) ${ }^{40}$. Given the nature of those attending the day hospital this is not surprising as a day hospital population predominantly consists of individuals experiencing or at risk of a decline in health or mobility. While subjective questionnaires are easy to administer, there are some limitations of PA questionnaires in this population, for example, successful tracking of PA activities and accurate recall over a specified time. Considering the levels of cognitive impairment found in this sample, PA questionnaires may not be the most accurate method to track PA levels at an individual level in day hospital attendees ${ }^{41}$. Physical accelerometers on the other hand have been shown to be increasingly accurate in identifying the PA estimation associated with daily living and can accurately track PA levels and SB in older adults (time spent, intensity and frequency) ${ }^{13}$. When measured objectively, the accelerometer derived information highlights the same concern as the IPAQ-E in relation to SB. Older adults in the UK have been shown, using an accelerometer, to spend approximately $80 \%$ of their days in $\mathrm{SB}^{10}$. In our study, a similar level of SB was found using both cutoff criteria, SB/Light PA accounted for $80-85 \%$ of their week. While the IPAQ-E and accelerometers reflect similar findings, the two tools were only moderately correlated with one another (Spearman R=0.3-0.59). For example, total time spent sitting per day was moderately correlated (negatively) with total time spent in MVPA across 7 days ( $R=$ -0.52). Given the moderate correlation between the IPAQ-E and physical accelerometers, physical accelerometers may offer an added benefit to clinicians in this current COVID-19 climate as it offers a means to track PA levels and response to interventions (in relation to SB) from a distance, limiting the requirement for close contact time.

Sarcopenia is an important consideration for all older adults, particularly those experiencing a change in their health or a functional decline. A key part of treatment of sarcopenia is exercise, particularly resistance exercise ${ }^{5}$. Physiotherapy has a key role in the delivery of exercise-based interventions to sarcopenic patients and in the education and facilitation of increasing PA levels while simultaneously reducing SB time. In this study, accelerometer tracked data indicated that sarcopenia (LL) was associated with increased amount of time in SB and low PA as well as reduced number of steps. Both aerobic base and resistancebased exercise have been shown to reduce sarcopenia prevalence and improve physical performance/muscular strength $^{42-43}$. Considering the patients' levels of frailty and multiple comorbidities, the identification of exercise that is safe and effective is imperative. Therefore, a simple method to increase an individual's PA level would be through increasing step count through accelerometer or pedometer monitoring. The monitoring of step counts can potentially be done using different tools, pedometers, and accelerometers or a smart phone if available. The relationship between reducing SB and increasing PA is important when reducing the risk of sarcopenia. Sanchez-Sanchez et al. ${ }^{13}$ identified that by decreasing SB by 1 hour and increasing MVPA by 1 hour led to a $50 \%$ reduction in sarcopenia risk in 5497 community dwelling older adults (Mean age $78.08 \pm S D=$ 5.7). The relationship between PA, SB and sarcopenia is well established but interventions can also be targeted for those at risk of sarcopenia or those with severe sarcopenia as per the EWGSOP2 criteria 5 . Unfortunately, due to the small sample size, further investigation into these smaller subgroups could not be conducted but highlights the potential for new areas of research in this patient cohort.

Another consideration for the use of accelerometers in this population group is the specific cut-off points to classify physical activity levels. In this study, two different cut-off scores for physical activity classification were used ('Freedson's Adult VM3 2011' from Actigraph \& SanchezSanchez et al. ${ }^{13}$ ). The two cut-offs were implemented as both cut-offs are validated using different groups (adults and older adults). Many authors have attempted to track PA levels of older adults using PA intensity categories based upon healthy young adults ${ }^{13}$. Using the two different cutoff criteria a difference of 7-8-hour difference in physical activity classification across a week was identified. Of the two databases used, the Sanchez-Sanchez et al. (2019) cutoffs were based upon an older adult while Freedsons' Adult VM3 2011 (from Actigraph database) is derived from 50 adults (Mean age 26.9 $\pm \mathrm{SD}=7.7$ ) walking on a treadmill at various speeds ${ }^{39}$. Different activities have different energy costs for younger and older adults. In Barnett et al. ${ }^{44}$, older adults (Mean age $70.2 \pm S D=7$ ), the MVPA walking cut point (Vertical axis 1013 CPM) as derived using an older adult population were substantially lower compared to the MVPA cut-off point based off a younger population group (Vertical axis 1952 CPM). In the systematic review of Gorman et al. ${ }^{45}$ (59 studies included), the cut points varied for MVPA from 574 to 3,250 CPMs, and for SB the cut-points varied from 50-500 CPMs. In relation to MVPA this can lead to variance ranging from 4-80 minutes a day spent in MVPA and 475 
to 665 minutes a day in $\mathrm{SB}^{45}$. The use of specific cut off points is important to identify and for the implementation of accelerometery in an older adult population. The use of specific cut-off points based upon older adults is important for accelerometers, when implementing their use in the management of sarcopenia. Clinicians should always look to use physical activity classification tables that are based upon the patient cohort that is being assessed and treated, like the classification of Sanchez-Sanchez et al. ${ }^{13}$. However, the findings of the current study highlight that the cut-offs of Sanchez-Sanchez et al. ${ }^{13}$ may be more applicable in this population group, but the authors cannot recommend a specific accelerometer physical activity classification table due to differences in sarcopenia assessment, our singlecentre design, and the limited sample size.

In this study, depending on whether sarcopenia was defined using grip strength or LL strength, the associations with accelerometer data differed. Grip strength has been recommended as a surrogate measure of $L L$ strength as they are highly correlated and handgrip strength is easier to measure in clinical practice ${ }^{46-48}$. In a similar older adult population, Yeung et al. ${ }^{49}$ and McGrath ${ }^{50}$, LL strength (knee extension) was found to have a higher association with health characteristics in comparison to handgrip strength while both strength measures were associated with a falls history, reduced gait speed, higher $A D L$ dependence, depression and nutrition. In our study, grip strength was only moderately associated with all physical performance measurement and LL strength $(R=0.3-0.59)$.

The variation in the strength of association between grip strength and LL strength is an important consideration for sarcopenia research. Fragala et al. ${ }^{51}$ identified both LL strength (isokinetic) and grip strength as predictors of slow gait, with LL strength a slightly stronger association compared to grip strength in two large datasets. Fragala et al. ${ }^{51}$ found that LL strength was a better predictor of gait speed, compared to grip strength, when a faster gait speed was reported (higher functioning population). This indicates that the association between grip strength and gait speed was less when the population had a higher gait speed/performed at a higher function. In addition, Fragala et al. ${ }^{51}$ highlighted that the relationship between grip strength and gait speed may be more attenuated in those with severe weakness. This is important to consider, given the accelerometer group were less frail and performed better at physical performance measures compared to the non-accelerometer group. It may be that grip strength may be more suitable assessment for older and less functional patients, where those younger and more functional will require a $L L$ strength test ${ }^{51}$. Another key factor to consider is that LL strength will play a more direct or fundamental role in a individual's capacity to engage with PA.

This is the first study to apply the EWGSOP2 criteria to older adults living in the community in an Irish day hospital setting and assess PA using physical accelerometers.
The findings highlight the feasibility of tracking PA using accelerometers in this population. Bias was minimised as much as possible, using a robust methodology and the use of standardised assessments and instructions. The study protocol was designed, with a key focus on reproducibility and transparency to enable future research to implement similar designs, to compare findings accurately. Due to the cross-sectional design of the study, no predictive factors of sarcopenia could be identified using the accelerometer derived information and due to limited resources (low sample size) a regression analysis could not be completed. Another limitation was the failure to reach the planned sample size of 180 (60 accelerometer participants). The final sample size of the study was 134 and 38 accelerometer participants, this was due to the closure of the RMDH due to COVID-19 pandemic in early March 2020. There are several limitations associated with the assessment procedure adopted. The study was limited to a small sample size due to limited resources of physical accelerometers. For BIA analysis, participants did not follow a strict fluid restriction for 24 hours prior to assessment or void bladder immediately prior. Given that BIA is influenced by fluid status, the lack of standardised procedure may have limited the accuracy of findings. There were significant differences between the accelerometer group and the main study group in relation to cognition, frailty, and physical performance measures. Measures were implemented to limit a selection bias as much as possible but more stringent measures may have been needed. Since cognition and age were significantly different between the accelerometer and non-accelerometer group, the feasibility of accelerometry in the older and more severe cognitively impaired cannot be concluded. We acknowledge that advanced age and cognitive impairment are challenges for this type of research that need alternative approaches ${ }^{52}$.

In conclusion, accelerometers are a feasible method to track PA and SB in older adults attending a day hospital service in Ireland. Step counts, time spent in PA and SB were associated with sarcopenia, however the association depended on the accelerometer cut points used and the muscle strength measurement used in the EWGSOP2 criteria. To appropriately manage sarcopenia, a greater understanding is required. Firstly, the assessment of sarcopenia needs be more consistent regarding the use of the EWGSOP2 guidelines, the assessment of muscle strength (grip or 5-STS or both) and muscle mass assessment. A more comprehensive and consistent measurement of sarcopenia using the EWGSOP2 will allow a greater understanding of sarcopenia in this population and enable research to progress. Once this issue of defining sarcopenia is addressed, further understanding regarding the application of accelerometer to aid track the efficacy of sarcopenia interventions. Potential future research can investigate the effectiveness of PA interventions and tracking accelerometer data over time to assess the influence on sarcopenia presence or adverse outcomes. In the meantime, the results of the present 
study underscore the practicality of the assessment of PA and SB in the geriatric day hospital setting using physical accelerometers. It is recommended that beyond research studies, the routine clinical assessment of sarcopenia and PA in the day hospital setting is incorporated as part of existing and future healthcare policies to improve outcomes for older adults.

\section{Funding}

The study was funded by an Irish Research Council Employment Based Postgraduate Programme Award (EBPPG/2019/50) in association with St James's Hospital. At the time of this submission RRO is funded by Science Foundation Ireland (SFI), grant number 18/FRL/6188.

\section{Ethics approval}

Ethical approval was granted by the Research Ethics Committee of SJH and Tallaght University Hospital (201910 List 39 [09]) in October 2019. The research Study was also approved by the St James's Hospital Research and Innovation department (Reference number: 5672).

\section{Consent to Participate}

Informed Consent (verbal and written) was obtained from all individual participants included in the study.

\section{Consent to Publish}

Participants signed informed consent regarding publishing their data (non-identifiable).

\section{Acknowledgements}

We wish to thank the study gatekeepers and all participants who took part in the study.

\section{References}

1. Marzetti E, Calvani R, Tosato M, Cesari M, Bari M, Cherubini A, Collamati A, D’Angelo E, Pahor M, Bernabei R, Landi F. Sarcopenia: an overview. Aging Clin Exp Res 2017;29(1):11-17.

2. Sayer A, Robinson S, Patel H, Shavlakadze T, Cooper C, Grounds M. New horizons in the pathogenesis, diagnosis and management of sarcopenia. Age and Ageing 2013;42(2): 145-150.

3. Witham M. Bridging the gap between the laboratory and the clinic for patients with sarcopenia. Biogerontology 2019;20(2):241-248.

4. Solane P, Marzetti E, Landi F, Zimmerman S. Understanding and Addressing Muscle Strength, Mass, and Function in Older Persons. JAMDA 2019;20(1): 1-4.

5. Cruz-Jentoft A, Bahat $G$, Bauer J, Boirie $Y$, Bruyère $O$, Cederholm T, Coope C, Landi F, Rolland Y, Sayer A, Schneider S, Sieber C, Topinkova E, Vandewoude M, Visser M, Zamboni M, Bautmans I, Baeyens J, Cesari M, Cherubini A, Kanis J, Maggio M, Martin F, Michel J, Pitkala K, Reginster J, Rizzoli R, Sánchez-Rodríguez D, Schols J. Sarcopenia: revised European consensus on definition and diagnosis. Age and Ageing 2019;48(1):16-31

6. Dos Santos L, Cyrino ES, Antunes M, Santos DA, Sardinha LB. Sarcopenia and physical independence in older adults: the independent and synergic role of muscle mass and muscle function. Journal of Cachexia Sarcopenia Muscle 2017;8(2):245-50.

7. Nascimento P, Poitras S, Bilodeau M. How do we define and measure sarcopenia? Protocol for a systematic review. Systematic Reviews 2018;7:51-60.

8. Donoghue O, O'Connell M. Research Brief: Physical Activity in Community-Dwelling Older Irish Adults. Dublin: The Irish Longitudinal Study on Ageing, 2016. URL (Accessed 9th September 2019): https://tilda.tcd.ie/publications/research-briefs/pdf/2016_ Research\%20Brief_Physical\%20Activity.pdf

9. Shaw S, Dennison E, Cooper C. Epidemiology of Sarcopenia: Determinants Throughout the Lifecourse. Calcif Tissue Int 2017; 101(3):229-247

10. Heesch K, Hill R, Aguilar-Farias N, Van Uffelen J, Pavey T. Validity of objective methods for measuring sedentary behaviour in older adults: a systematic review. International Journal of Behavioral Nutrition and Physical Activity 2018;15(1):119-136.

11. Ryu M, Jo J, Lee Y, Chung YS, Kim KM, Baek WC. Association of physical activity with sarcopenia and sarcopenic obesity in community-dwelling older adults: the Fourth Korea National Health and Nutrition Examination Survey. Age and Ageing 2013;42(6): 734-740

12. Steffl M, Bhannon R, Sontakova L, Tufano J, Shiells K, Holmerova I. Relationship between sarcopenia and physical activity in older people: a systematic review and meta-analysis. Clinical Interventions in Aging 2017;12:835-845.

13. Sanchez-Sanchez J, Manas A, Garcia-Garcia F, Ara I, Carnicero J, Walter S, Rodriguez-Manas L. Sedentary behaviour, physical activity, and sarcopenia among older adults in the TSHA: isotemporal substitution model. Journal of Cachexia, Sarcopenia and Muscle 2019;10(1):188-198.

14. Nascimento CM, Ingles M, Salvador-Pascual A, Cominetti MR, Gomez-Cabrera MC, Vina J. Sarcopenia, frailty and their prevention by exercise. Free Radical Biology and Medicine 2019;132: 42-49.

15. Morley J. Frailty and Sarcopenia: The New Geriatric Giants. Revista de investigacion clinica; organo del Hospital de Enfermedades de la Nutricion 2016:68(2):59-67

16. Shephard RJ. Limits to the measurement of habitual physical activity by questionnaires. British Journal of Sports Medicine 2003;37:197-206

17. Doherty A, Jackson D, Hammeria N, Plotz T, Olivier P, Granat M, White T, Van Hees V, Trenell M, Owen C, Preece S, Gillions R, Sheard S, Peakman T, Brage S, Wareham N. Large Scale Population Assessment of Physical Activity Using Wrist Worn Accelerometers: The UK Biobank Study. PLoS ONE 2017;12(2):eO 169649.

18. National Clinical Programme for Older People. Specialist Geriatric Services Model of Care'. Health Service Executive- National Clinical Programme for Older people committee 2012. URL (Accessed 5th Feb 2020): https://www.hse.ie/eng/services/publications/clinicalstrategy-and-programmes/specialist-geriatric-services-model-ofcare.pdf

19. O'Caoimh R, Kennelly S, O'Shea D. Can the Geriatric Day Hospital Act As a Hub for Services for Older People across the Spectrum of Ageing from Active Ageing to Advanced Frailty? Frontiers in Medicine 2018;5(23): 1-4.

20. Lekan D. Frailty and Other Emerging Concepts in Care of the Aged Southern Online Journal of Nursing Research 2009;9(2): 1-29.

21. Palmer K, Monaco A, Kivipelto M, et al. The potential long-term impact of the COVID-19 outbreak on patients with non-communicable diseases in Europe: consequences for healthy ageing. Aging Clin Exp Res 2020;32(7): 1189-1194.

22. Parmelee PA, Thuras PD, Katz IR, Lawton MP. Validation of the Cumulative IIIness Rating Scale in a geriatric residential population. $J$ Am Geriatr Soc 1995;43(2):130-137.

23. Salvi F, Miller M, Grilli A, Giorgi R, Towers A, Morichi V, Spazzafumo L, Mancinelli L, Espinosa E, Rappelli A, Dessi-Fulgheri P. A Manual of 
Guidelines to Score the Modified Cumulative IIIness Rating Scale and Its Validation in Acute Hospitalized Elderly Patients. J Am Geriatr Soc 2008;56(10): 1926-1931.

24. Roberts H, Denison H, Martin H, Patel H, Syddall H, Cooper C, Sayer A. A review of the measurement of grip strength in clinical and epidemiological studies: towards a standardised approach. Age and Ageing 201 1;40(4):423-429

25. Guralnik J, Simonsick E, Ferrucci L, Glynn R, Berkman L, Blaer D, Scherr P, Wallace R. A Short Physical Performance Battery Assessing Lower extremity function: Association with Self-reported Disability and prediction of Mortality and Nursing Home Admission. Journal of Gerontology: Medical Sciences 1994;49(2):85-94.

26. Sergi G, De Rui M, Veronese N, Bolzetta F, Berton L, Carraro S, Bano G, Coin A, Manzato E, Perissinotto E. Assessing appendicular skeletal muscle mass with bioelectrical impedance analysis in free-living Caucasian older adults. Clinical Nutrition 2015;34(4):667-673.

27. Scafoglieri A, Clarys JP, Bauer J, Verlaan S, Van Malderen L, Vantieghem S, Cederholm T, Sieber C, Mets T, Bautmans I. Predicting appendicular lean and fat mass with bioelectrical impedance analysis in older adults with physical function decline The PROVIDE study. Clinical Nutrition 2017:36(3):869-875.

28. Viet L, Verschuren, M. Measurement protocols. The Feasibility of a European Health Examination Survey (FEHES) Recommendations, 2008. URL (Accessed August 2019): https://ec.europa.eu/health/ ph_information/dissemination/reporting/docs/fehes_protocols_ en.pdf

29. Herman T, Giladi N, Hausdorff JM. Properties of the 'timed up and go' test: more than meets the eye. Gerontology 2011;57(3):203-210.

30. Malmstrom TK, Morley JE. SARC-F: a simple questionnaire to rapidly diagnose sarcopenia. J Am Med Dir Assoc 2013; 14(8):53 1-532.

31. Kaiser M, Uter W, Bauer J, Guigoz Y. Validation of the Mini Nutritional Assessment Short-Form (MNA(r)-SF) A practical tool for identification of nutritional status. Journal of Nutrition Health and Aging 2009; 13(9): 782-790

32. Glynn K, Coen R, Lawlor B. Is the Quick Mild Cognitive Impairment Screen (QMCl) more accurate at detecting mild cognitive impairment than existing short cognitive screening tests? A systematic review of the current literature. International Journal of Geriatric Psychiatry 2019;34(12):1739-1746.

33. Rockwood K, Song X, MacKnight C, et al. A global clinical measure of fitness and frailty in elderly people. CMAJ 2005; 173(5):489-495.

34. Romero-Ortuno R, O'Shea D, Kenny RA. The SHARE Frailty Instrument for primary care predicts incident disability in a European populationbased sample. Primary Care 201 1;19(5):301-309.

35. Sjóstróm M, Ainsworth BE, Bauman A, Bull F, Hamilton-Craig CR, Sallis JF. Guidelines for Data Processing Analysis of the International Physical Activity Questionnaire (IPAQ) - Short and Long Forms, 2009. URL (Accessed August 2019): https://sites.google.om/site/ theipaq/scoring-protocol.

36. Knaier R, Höchsmann C, Infanger D, Hinrichs T, Schmidt-Trucksäss A. Validation of automatic wear-time detection algorithms in a freeliving setting of wrist-worn and hip-worn ActiGraph GT3X. BMC Public Health 2019; 19(1):244

37. Choi L, Ward SC, Schnelle JF, Buchowski MS. Assessment of wear/ nonwear time classification algorithms for triaxial accelerometer. Med Sci Sports Exerc 2012;44(10):2009-2016.

38. Schrack JA, Cooper R, Koster A, etal. Assessing Daily Physical Activity in Older Adults: Unraveling the Complexity of Monitors, Measures, and Methods. J Gerontol A Biol Sci Med Sci. 2016;71(8):1039-1048.

39. Sasaki JE, John D, Freedson PS. Validation and comparison of ActiGraph activity monitors. Journal of Science and Medicine in Sport 2011;14(5):411-416.

40. Kandola A, Stubbs B, Koyanagi A. Physical multimorbidity and sedentary behavior in older adults: Findings from the Irish longitudinal study on ageing (TILDA). Maturitas 2020;134:1-7.

41. Cleland C, Ferguson S, Ellis G, Hunter RF. Validity of the International Physical Activity Questionnaire (IPAQ) for assessing moderateto-vigorous physical activity and sedentary behaviour of older adults in the United Kingdom. BMC medical research methodology 2018;18(1):176.

42. Bauer J, Morley J E, Schols A, Ferrucci L, Cruz-Jentoft AJ, Dent E, Baracos VE, Crawford JA, Doehner W, Heymsfield SB, Jatoi A, Kalantar-Zadeh K, Lainscak M, Landi F, Laviano A, Mancuso M, Muscaritoli M, Prado CM, Strasser F, von Haehling S, Anker SD. Sarcopenia: A Time for Action. An SCWD Position Paper. Journal of Cachexia, Sarcopenia and Muscle 2019;10(5):956-961.

43. Saggini R, Carmignano SM, Cosenza LC, Palermo T, Bellomo RG. Chapter 9 Sarcopenia in Chronic IIIness and Rehabilitative Approaches. Frailty and Sarcopenia- Onset, Development and Clinical Challenges; 2017. URL (Accessed March 2020): https://www.intechopen. com/books/frailty-and-sarcopenia-onset-development-andclinical-challenges/sarcopenia-in-chronic-illness-and-rehabilitativeapproaches

44. Barnett A, van den Hoek D, Barnett D, Cerin E. Measuring moderateintensity walking in older adults using the ActiGraph accelerometer. BMC geriatrics 2016;16(1):211

45. Gorman E, Hanson H, Yang PH, Khan KM, Liu-Ambrose T, Ashe MC. Accelerometry analysis of physical activity and sedentary behavior in older adults: a systematic review and data analysis. European Review of Aging and Physical Activity: Official Journal of the European Group for Research into Elderly and Physical Activity 2014;1 1(1):35-49.

46. McGrath R. Understanding the Feasibility and Validity of Muscle Strength Measurements in Aging Adults. JAMDA 2019;20(1):99100.

47. Newman A, Kupelian V, Visser M, Simonsick E, Goodpaster B, Nevitt M, Kritchevsky S, Tylavsky F, Rubin S, Harris T. Sarcopenia: Alternative Definitions and Associations with Lower Extremity Function. J Am Geriatr Soc 2003;51(1 1): 1602-1609

48. Beaudart C, Rolland Y, Cruz-Jentoft AJ, Bauer JM, Sieber C, Cooper C, Al-Daghri N, Araujo de Carvalho I, Bautmans I, Bernabei R, Bruyère O, Cesari M, Cherubini A, Dawson-Hughes B, Kanis JA, Kaufman JM, Landi F, Maggi S, McCloskey E, Petermans J, Fielding RA. Assessment of Muscle Function and Physical Performance in Daily Clinical Practice: A position paper endorsed by the European Society for Clinical and Economic Aspects of Osteoporosis, Osteoarthritis and Musculoskeletal Diseases (ESCEO). Calcified Tissue international 2019; 105(1): 1-14.

49. Yeung SS, Reijnierse EM, Trappenburg MC, Blauw GJ, Meskers CG, Maier AB. Knee extension strength measurements should be considered as part of the comprehensive geriatric assessment. BMC Geriatrics 2018; 18(130): 1-8.

50. McGrath R, Johnson N, Klawitter L, Mahoney S, Trautman K, Carlson C, Rockstad, E, Hackney KJ. What are the association patterns between handgrip strength and adverse health conditions? A topical review. SAGE Open Medicine 2020;8:20503 12120910358.

51. Fragala MS, Alley DE, Shardell MD, Harris TB, McLean RR, Kiel DP, Cawthon PM, Dam TT, Ferrucci L, Guralnik JM, Kritchevsky SB, Vassileva MT, Gudnason V, Eiriksdottir G, Koster A, Newman A, Siggeirsdottir K, Satterfield S, Studenski SA, Kenny AM. Comparison of Handgrip and Leg Extension Strength in Predicting Slow Gait Speed in Older Adults. Journal of the American Geriatrics Society 2016; 64(1):144-150.

52. De Cock AM, Perkisas S, Verhoeven V, Vandewoude M, Fransen E, Remmen R. The impact of cognitive impairment on the physical ageing process. Aging Clin Exp Res 2018;30(1 1):1297-1306. 


\section{Appendix 1.}

\section{Accelerometer Descriptors}

\begin{tabular}{|c|c|}
\hline Variable & Description \\
\hline Sedentary Bout & Reduced activity which equates to $>10$ minutes of registering $<99$ CPM \\
\hline Total Sedentary Bouts & Number of sedentary bouts detected in the dataset \\
\hline Total Length of Sedentary Bouts & The total sedentary time detected in the entire dataset \\
\hline Max Length of Sedentary Bouts & The length of the longest sedentary bout in the entire dataset \\
\hline Min Length of Sedentary Bouts & The length of the shortest sedentary bout in the entire dataset \\
\hline Daily Average of Sedentary Bouts & The total length of sedentary bouts divided by the total valid days in the dataset \\
\hline Sedentary Break & $\begin{array}{l}\text { Breaks in sedentary time were calculated as any interruption in sedentary time lasting one minute } \\
\text { or longer in which the accelerometer counts per minute rose to or above } 100 \mathrm{CPM}\end{array}$ \\
\hline Total Sedentary Break & The total number of breaks in sedentary activity for the entire dataset \\
\hline Total Length of Sedentary Breaks & The sum of all the times between sedentary bout sin the dataset \\
\hline Average Length of Sedentary Breaks & $\begin{array}{l}\text { An average of all of the times between sedentary bouts (total length of sedentary breaks/total } \\
\text { sedentary breaks) }\end{array}$ \\
\hline Max Length of Sedentary Breaks & The length of the longest sedentary break in the entire dataset \\
\hline Minimum Length of Sedentary Breaks & The length of the shortest sedentary break in the entire dataset \\
\hline Daily Average of Sedentary Breaks & The total length of sedentary breaks divided by total valid days in the dataset \\
\hline Step Count Max & The highest number of steps in one epoch in an hour for the specified day \\
\hline Lux Count & Measure of ambient light \\
\hline MVPA & Moderate and Vigorous Physical Activity combined \\
\hline \%MVPA & $\begin{array}{l}\text { The percentage of total time (wear-time only if "exclude non-wear." is checked) spent at or above } \\
\text { the MVPA cut point level }\end{array}$ \\
\hline Average MVPA & The time in MVPA divided by calendar days of valid days \\
\hline Freedson Bout & $\begin{array}{l}\text { Exercise meeting a certain criterion lasting at least } 10 \text { minutes long with min counts of } 1952 \text { CPM, } \\
\text { Max CPM of } 50000 \text { (unreachable), with a drop time of } 2 \text { minutes }\end{array}$ \\
\hline Drop Time & $\begin{array}{l}\text { Drop time acts as a tolerance, once a bout is detected (CPM >1952), the bout is allowed to } \\
\text { experience no more than a } 2 \text {-minute drop outside the minimum count level. }\end{array}$ \\
\hline
\end{tabular}

